\title{
Effects of combined digital inclusion and physical activity intervention on the cognition of older adults in Brazil
}

\begin{abstract}
Ana Paula Vicentin $\mathrm{PhD}^{\mathrm{a}}$, Eduardo Jardim Simões MD MSc MPH ${ }^{\mathrm{b}}$, Ana Claudia Bonilha $\mathrm{MSc}^{\mathrm{a}}$, Claudine Lamoth $\mathrm{PhD}^{\mathrm{c}}$, Solange Andreoni $\mathrm{PhD}^{\mathrm{d}}$, Luiz Roberto Ramos MD PHD ${ }^{\mathrm{d}, *}$

aProgram of Post-Graduation in Community Health, Department of Preventive Medicine, Universidade Federal de Sao Paulo (UNIFESP), São Paulo, Brazil; ${ }^{b}$ Department of Health Management and Informatics, School of Medicine, University of Missouri, Columbia, Missouri, USA; ${ }^{c}$ Center for Human Movement Sciences, University Medical Centre, University of Groningen, Groningen, Netherlands; ${ }^{d}$ Department of Preventive Medicine, Escola Paulista de Medicina, Universidade Federal de Sao Paulo (UNIFESP), Sao Paulo, Brazil; ${ }^{*}$ Corresponding author: Irramos1953@gmail.com
\end{abstract}

\begin{abstract}
Background: Cognitive decline in normal aging is a major concern in aging societies as it impacts independence in daily life, quality of life and life expectancy. Population aging in the coming decades makes the prevention of cognitive decline and maintenance of functional capacity, important public health issues due to the social impact and economic costs of the demographic process.

Objective: Research aims to evaluate the effectiveness of combined digital inclusion and physical activity interventions in the prevention of cognitive and functional loss among elderly residents in a large urban center in Brazil. The study is a comparative controlled study in primary care setting.

Methods: We sampled 112 individuals from a population cohort of adults ages 60 and older, previously evaluated by Clinical Dementia Rating scale (zero and 0.5 scores), without depressive symptoms allocated to intervention group (IG) and control group (CG). IG participants attended a computer-based digital inclusion program combined with physical activity, with 80-min sessions twice a week for 17 weeks, whereas the CG followed their daily routine. We administered neuropsychological tests to all participants at baseline and follow-up (Montreal Cognitive Assessment - MoCA, Mini-Mental Status Exam - MMSE, list of words, verbal fluency, and animal categories), plus an evaluation of independence in activities of daily living - ADL and the Geriatric Depression Scale - GDS.

Results: An intention-to-treat analysis showed an average increase of 1.23 points in MoCA scores after the intervention $(p=0.012)$. Secondary analysis considering the number of sessions attended showed that MoCA scores increased 0.074 points on average for each session attended $(p<0.001)$ and MMSE scores increased 0.029 points on average for each session attended $(p<0.022)$. There were no statistical differences post-intervention for the other variables.

Conclusions: The intervention improved global cognition among elderly residents. The results did not show improvements in specific cognitive domains or independence in daily life. We recommend longer and more powerful studies statistically to further assess the impact of this strategy in the prevention of functional and cognitive loss.
\end{abstract}

Keywords: digital inclusion; cognitive intervention; physical activity intervention; controlled trial, older adults

\section{INTRODUCTION}

Cognitive decline in normal aging is a major concern in aging societies. It impacts on learning, memory, language, and orientation, therefore impairing independence in daily life activities as well as the quality of life and life expectancy (Ramos et al., 2001, Singh-Manoux et al., 2012; Small \& Bäckman, 1999). Population aging in the coming decades makes the prevention of cognitive decline and maintenance of independence important issues in public health, due to the social impact and economic costs (Prince et al., 2015). This scenario is even more stringent in low and middle-income countries where age-related cognitive decline is aggravated by low educational levels and poor health status (Nitrini et al., 2009, Prince et al., 2014). Primary prevention should focus on reducing modifiable risk factors for cognitive impairment, such as physical inactivity, depression, and low educational at- 
tainment, among others (Livingston et al., 2017).

There are evidences that cognitive stimulation programs can slow down cognitive decline in old age. They include cognitive training with a set of exercises involving repeated practice and increased difficulty in levels of cognitive functions (Bamidis et al., 2014, Clare \& Woods, 2003; Martin et al., 2011). Unlike cognitive rehabilitation, cognitive stimulation/training does not emphasize a specific cognitive domain but includes procedures designed to provide general cognitive stimulation, in a social context, presenting the benefits of community-based programs (Clare \& Woods, 2003).

The use of technology is nowadays part of the daily life of people all over the world. The information society might have potential benefits to the quality of life for older people, as it enables them to establish and maintain social relations, protecting them from social isolation ( $\mathrm{Boz} \&$ Karatas, 2015). Indeed, technological devices are frequently being used to design new intervention programs for cognitive stimulation of older adults in the community (Klusmann et al., 2010; Kueider, Parisi, Gross, \& Rebok, 2012; Lampit, Hallock, \& Valenzuela, 2014; Shao et al., 2015). Technological devices, however, may present a challenge for those lacking previous experience with them. For this population, it's necessary to previously develop the ability to use computers and the internet, promoting digital inclusion and digital literacy (Lankshear \& Knobel, 2008).

A nationwide cohort of people aged 50 and older in England, found evidences that Internet/email use reduced cognitive decline and increased their delayed recall capability, with a strong effect size compared with non-users and intermittent users (Xavier et al., 2014). D’Orsi et al. (2017) found that baseline internet use was associated with a $40 \%$ reduction in dementia risk assessed after six years. It also reduced the incidence of IADL impairment (D'Orsi et al., 2014) and made users more likely to report healthy behaviors than 'never users' (Xavier et al., 2013).

Results of community intervention studies, however, are inconsistent; the effectiveness of digital inclusion to enhance cognitive function remains unclear and design aspects, such as length (minutes per session), frequency (sessions per week), and duration (number of weeks) seem to play a role in the results (Lampit et al., 2014; Shao et al., 2015). Worth mentioning that the majority of the studies took place in high-income countries - USA and Europe (Law et al., 2014). Social determinants in low-middle-income countries such as restricted access to formal schooling may influence the results of interventions (Scazufca et al.,
2008). A recent systematic review was inconclusive on whether computerized cognitive training can produce lasting effects and suggested more research to find out whether longer periods of training work better (Gates et al. 2019).

A systematic review showed that combined cognitive and exercise training in older adults with or without cognitive impairment can be effective for improving cognitive function and functional status (Law et al., 2014). A recent meta-analysis with more than 2600 subjects over 50 years of age without known cognitive impairments, from 20 studies mostly performed in high-income countries, showed a small overall effect size in the cognition of combined intervention versus a control group. Greater improvements were seen among the older age groups compared to the younger ones. Group settings appeared to be more beneficial than home-based individual programs (Zhu et al., 2016).

We thus aimed to examine the effects of a controlled intervention that combined digital inclusion and physical activity on the cognitive function and independence in daily life of a community-based cohort of elders in Brazil.

\section{Methods \\ Study area and population}

Between December 2007 and April 2008, we drew a random sample of 2000 people aged 60 years or more living in a middle-class neighbourhood, with high educational level and low migration rates, in the city of São Paulo for household survey to assess functional capacity (Ramos et al., 2013). After the home interview we invited all interviewees to participate in an open cohort and a total of 1155 elderly attended and had a baseline gerontological evaluation at the university aging centre (CSA) that provided data on socio-demographic characteristics, referred chronic diseases, medications in use, cognitive status, and independence in daily life.

\section{Study sample}

In 2015, we contacted a sub-sample of this cohort, previously evaluated for dementia using a validated Portuguese version of the Clinical Dementia Rating scale (CDR) (Montaño \& Ramos, 2005). Between October 2015 and March 2016 all subjects were contacted and invited to participate in the present study, after an explanatory talk. Those who agreed were booked for a new application of the CDR, applied by a trained professional through an unstructured interview with a relative or close contact of the participant. The inclusion criterion was being part of the aged cohort $(60+)$ in the study area (Ramos et al. 2001), with normal cognition $(C D R=0)$ or mild cognitive impairment $(\mathrm{CDR}=0.5)$ (Morris, 
1996; Montaño \& Ramos, 2013). The exclusion criterion was the diagnosis of a chronic or debilitating health condition that impeded a subject to participate in either intervention activities. There are evidences that cognitive stimulation programs can slow down cognitive decline in old age. After signing the consent form, subjects were assigned to two comparative study groups - intervention and control - with an allocation ratio of 1:1, based on the sequence of their arrival in the study. Within the limitation of the cohort sub-sample size, we also frequency-matched intervention group subjects to a control group with respect to gender (M:F).

\section{Outcome measures}

Neuropsychological tests were administered at baseline and after the intervention. The Montreal Cognitive Assessment (MoCA) (Nasreddine, Phillips, \& Bedirian, 2005) and Mini Mental State Examination (MMSE) (Folstein et al., 1975) were used to assess global cognition. At the inception of the cohort, subjects answered the MMSE. After the first wave, and before the start of the present study, we decided to use the MoCA because it has shown less educational bias, but we kept the MMSE for the sake of longitudinal comparisons. Both instruments have a maximum score of 30 points indicating intact cognition, determined by the sum of points in different cognitive domains.

The Word List Memory composed of twelve unrelated words was used to assess memory; the score is obtained by the sum of the words evoked in three trials, with a maximum score of 36 points (Atkinson\& Shiffrin, 1971). Verbal Fluency was assessed by the animal categorical test (Isaacs \& Kennie, 1973). Independence in daily life was measured by an Activities of Daily Living (ADL) scale that assessed limitations to perform 15 personal and instrumental $A D L$, through a self-reported questionnaire (BOMFAQ) (Ramos, 2003) that generates a zero to 15 score, in which higher scores indicate more limitations and less independence.

The validated Portuguese version of the Geriatric Depression Scale (GDS) (Paradela, Lourenço, \& Veras, 2005) was used to detect the presence of clinically relevant symptoms of depression (Yesavage et al., 1982). Although we considered depressive symptoms an exclusion criterion, they were also an outcome variable, considering that only people without depressive symptoms were included in the study.

\section{Intervention and control groups}

The intervention group (IG) participants were supposed to attend 34 sessions (two/week) at the CSA, each group with seven participants and three trained assistants present. The sessions included an initial 20 minutes of physical activ- ity - global stretching and light aerobic exercises in the surroundings. Next, we offered the participants 40-45 minutes of computer activities focusing on digital inclusion and use of the internet, followed by 15-20 minutes of group discussion about what they experienced in the sessions. The content covered in computer sessions followed a gradual sequence of difficulty, which allows the exploration of the machine focusing on digital inclusion. The protocol had 4 levels: first, development of psychomotricity, involving the use of a mouse, keyboards, virtual keyboard, drawing creation, photo galleries, PowerPoint presentations; second, identity, viewer emergence as you explore various devices and interactive tasks based on internet browsing and individual games; third self-awareness, criticism, and accountability in the use of devices and interactive tasks using search engines, blogs, quiz games, virtual games and fourth, alterity, autonomy and cooperation, ending with the use of electronic mail, Skype, Social Networks, cooperative games (Xavier, 2004; Xavier et al., 2008). The hardware used were: keyboards, mice, webcams, microphones, 17" monitors. Experienced software were: Windows $\AA$, Skype $\AA$, Internet browsers, Webmail, Messenger $\AA$, Google $\AA$

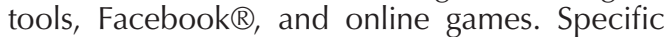
workshops dealt with the handling of smartphones. All the computer sessions had graduate students as monitors to facilitate subjects learning. This intervention methodology was based on previous studies (Krug et al., 2015; Krug et al., 2017, Xavier et al., 2004). The control group (CG) followed their daily domestic routine and had medical follow-up offered at CSA, the same as the IG. We re-evaluated both groups 17 weeks after baseline.

\section{Data analysis}

Differences in baseline between intervention and control groups for all the explanatory variables were analyzed through Student's t-tests for independent samples and Fischer's tests for categorical variables. We implemented a multivariate linear regression to analyze the associations of outcomes and the intervention. For each outcome, we adjusted the regression model effects of interventions to other explanatory variables and baseline measures of the outcomes variables.

An intention to treat (ITT) principle was used with the inclusion of all participants who presented a follow-up measure of the outcome, irrespective of deviations from treatment protocol (e.g., non-compliance). Dependent variables in the models included the follow-up measure of the response variables of interest (MoCA, MMSE, word list, evocation, verbal fluency, GDS, and ADL) as dependent variables. The explanatory variables were gender (male and female), age in 
Table 1. Baseline characteristics of Intervention Group and Control Group - categorical variables.

\begin{tabular}{lcccccccc}
\hline & & \multicolumn{2}{c}{$\begin{array}{c}\text { Total } \\
(\mathbf{n = 1 0 7})\end{array}$} & $\begin{array}{c}\text { Intervention group } \\
(\mathbf{n = 5 3 )}\end{array}$ & $\begin{array}{c}\text { Control group } \\
(\mathbf{n = 5 4 )}\end{array}$ & p-value* \\
\hline \multirow{3}{*}{ Gender } & & $\mathrm{n}$ & $\%$ & $\mathrm{n}$ & $\%$ & $\mathrm{n}$ & $\%$ & \\
& Male & 21 & 19.6 & 13 & 24.5 & 8 & 14.8 & 0.232 \\
& Female & 86 & 80.4 & 40 & 75.5 & 46 & 85.2 & \\
\multirow{2}{*}{ Education } & Low & 4 & 3.7 & 1 & 1.9 & 3 & 5.6 & \\
& Medium & 18 & 16.8 & 9 & 17.0 & 9 & 16.7 & 0.785 \\
& High & 85 & 79.4 & 43 & 81.1 & 42 & 77.8 & \\
\hline \multirow{2}{*}{ CDR status } & 0 & 56 & 52.3 & 31 & 58.5 & 25 & 46.3 & 0.247 \\
& 0.5 & 51 & 47.7 & 22 & 41.5 & 29 & 53.7 & \\
\hline
\end{tabular}

${ }^{*} p$ value is based on Fisher's test; $C D R=C$ linical Dementia Rating.

years, schooling (low as less than 4 years in formal school, medium as 4 to 8 years, and high as 8 or more years), CDR status (zero or 0.5 ), and group insertion (intervention or control). The explanatory variables age and baseline measures of the outcome variables were centered at their means at baseline in order to make the intercept of the regression equations interpretable. We used a backward procedure for the removal of non-significant explanatory variables at a 0.05 level to obtain a final model.

In a second analysis, considering that the subjects in the IG presented different rates of adherence to activities, we used the number of attended interventions as the main independent variable. As some subjects in the IG did not attend any sessions, the IG indicator coefficients would account for the change in constants (intercepts) due to the zero attended sessions in the IG. As in the previous analysis, we used the backward procedure for the removal of non-significant explanatory variables from the final model.

Interactions among the explanatory variables were not found any in either analysis. We also report effect sizes (measured by partial $\eta^{2}$ ) and the power $(\%)$ to detect the differences from zero of the regression coefficients. Norms for interpreting $\eta^{2}$ are: $0.01=$ small effect, $0.06=$ moderate effect, and $0.14=$ large effect. All statistical analyses were performed with SPSS 24.0 (IBM Corp., Chicago, IL).

\section{Ethical aspects}

The Ethics Research Committee of the Universidade Federal de São Paulo approved all procedures (Process no. 1.232.164, project 0960/2015). All participants signed the informed consent form.

Table 2. Baseline characteristics of Intervention group and Control group - continuous variables.

\begin{tabular}{lccccccc}
\hline & \multicolumn{2}{c}{$\begin{array}{c}\text { Total } \\
(\mathbf{n = 1 0 7})\end{array}$} & \multicolumn{2}{c}{$\begin{array}{c}\text { Intervention group } \\
(\mathbf{n = 5 3 )}\end{array}$} & $\begin{array}{c}\text { Control group } \\
(\mathbf{n = 5 4})\end{array}$ & p-value* \\
\hline & Mean & SD & Mean & SD & Mean & SD & \\
\hline Age (years) & 75.8 & 7.0 & 76.3 & 6.8 & 75.3 & 7.2 & 0.461 \\
MoCA & 22.6 & 3.6 & 22.7 & 3.2 & 22.6 & 3.9 & 0.796 \\
MMSE & 27.1 & 2.4 & 27.1 & 2.5 & 27.1 & 2.3 & 0.997 \\
GDS score & 1.9 & 2.1 & 1.7 & 2.1 & 2.1 & 2.0 & 0.239 \\
Word list & 18.1 & 5.5 & 17.6 & 5.5 & 18.7 & 5.6 & 0.315 \\
Evocation & 6.5 & 2.6 & 6.5 & 2.7 & 6.5 & 2.6 & 0.957 \\
Verbal fluency & 5.3 & 2.0 & 5.2 & 2.1 & 5.3 & 1.9 & 0.858 \\
ADL & 1.9 & 2.7 & 1.5 & 2.10 & 2.3 & 3.3 & 0.170 \\
\hline
\end{tabular}

${ }^{*} \mathrm{p}$-values are based on t test for independent samples.

MoCA=Montreal Cognitive Assessment; MMSE=Mini Mental State Examination; GDS=Geriatric Depression Scale; $\mathrm{ADL}=$ activities of daily living. 
Table 3. Initial and final model comparing effects of the interventions after 4 months on MoCA, modified ITT.

\begin{tabular}{|c|c|c|c|c|c|c|c|}
\hline $\begin{array}{l}\text { Dependent } \\
\text { variable }\end{array}$ & $\begin{array}{l}\text { Independent } \\
\text { variable }\end{array}$ & B & $95 \% \mathrm{Cl}$ & $\mathbf{t}$ & $\mathbf{p}$ & $\begin{array}{c}\text { Partial } \eta^{2} \\
(\%)\end{array}$ & $\begin{array}{c}\text { Power } \\
(\%)\end{array}$ \\
\hline Initial model & Constant* & 23.5 & {$[22.0 ; 25.0]$} & 31.7 & $<0.001$ & & \\
\hline \multirow[t]{7}{*}{ MoCA } & Intervention group & 1.3 & {$[0.3 ; 2.2]$} & 2.6 & 0.010 & 6.5 & 73.6 \\
\hline & MoCA baseline ct & 0.6 & {$[0.4 ; 0.8]$} & 7.6 & $<0.001$ & 37.1 & 100.0 \\
\hline & Female gender & 1.0 & {$[-0.3 ; 2.3]$} & 1.6 & 0.123 & 2.4 & 33.8 \\
\hline & Low schooling & -2.4 & {$[-5.1 ; 0.3]$} & -1.8 & 0.076 & 3.1 & 42.7 \\
\hline & Medium schooling & -0.3 & {$[-1.7 ; 1.2]$} & -0.4 & 0.731 & 0.1 & 6.4 \\
\hline & CDR 0.5 & -0.3 & {$[-1.3 ; 0.7]$} & -0.5 & 0.600 & 0.3 & 8.2 \\
\hline & Age ct & -0.04 & {$[-0.11 ; 0.03]$} & -1.1 & 0.256 & 1.3 & 20.5 \\
\hline$\overline{\text { Final model }}$ & Constant* & 24.1 & {$[23.4 ; 24.7]$} & 71.2 & $<0.001$ & & \\
\hline \multirow[t]{2}{*}{$\mathrm{MoCA}$} & Intervention group & 1.2 & {$[0.3 ; 2.2]$} & 2.6 & 0.012 & 6.0 & 72.1 \\
\hline & MoCA baseline ct & 0.7 & {$[0.6 ; 0.8]$} & 10.3 & $<0.001$ & 50.2 & 100.0 \\
\hline
\end{tabular}

*Reference: control group, male gender, high schooling, ct=centered at baseline mean value. Cl: confidence interval; MoCA: Montreal Cognitive Assessment; ITT: Intention to treat.

\section{Results}

At the beginning of the study, 164 subjects were contacted, 13 declined to participate and 39 did not meet the inclusion criteria (no subjects were excluded from the initial sample because of the exclusion criterion), resulting in 112 participants, 56 in the Intervention Group (IG) and 56 in the Control Group (CG).

In the IG, three participants were not included in the analyses (one died, one moved to another city, and one had a fracture in the upper limb post-evaluation). The number of attended sessions in the intervention group ranged from zero to 33 (mean=19.2, SD=11.2, median=25). Six subjects in the intervention group did not attend any sessions but performed the final assessments. All subjects in the IG did not attend at least one session of the planned 34 intervention sessions. In the CG, two participants did not respond to the call for post evaluation.

Finally, there were 53 older adults in the IG, $24.5 \%$ men $(n=13)$, and $75.5 \%$ women $(n=40)$, with a mean age of $76.3 \pm 6.8$ years. In the CG, there were 54 participants, including $14.8 \%$ men $(n=8)$ and $85.2 \%$ women $(n=46)$, with a mean age of $75.3 \pm 7.2$ years. No significant differences were found in baseline means of the characteristics between intervention and control groups (Tables 1 and 2).

The IG showed a significantly higher MoCA mean score after 4 months by 1.23 points $(p=0.012)$ than the CG. No significant differences were found after 17 weeks for MMSE, GDS, Word List, Evocation, Verbal Fluency, and ADL in the IGvs.CG after adjusting for the baseline measurements of outcomes, gender, CDR status, schooling, and age. The results from the regression models for the final response variables in the ITT analyses are presented in Table 3.

Secondary analyses assessed the contribution of the number of sessions the volunteers participated in ( 0 to a maximum of 33) to the final response variables. The number of attended sessions was an independent predictor of final MoCA and MMSE scores. Final MoCA scores increased on average by 0.07 points $(p<0.001)$ for each one unit of increase in the number of attended sessions. Final MMSE scores increased on average by 0.03 points $(p=0.022)$ per unit increase in the number of attended sessions. The number of attended sessions did not significantly explain final scores of GDS, Word List, Evocation, Verbal Fluency, or ADL after adjusting for their baseline measurements, and baseline measures of gender, initial CDR status, schooling, and age. Again, schooling level played a significant role in MMSE and Verbal Fluency mean scores. As in the analysis that followed the ITT principle, age had a significant effect on Word List and Evocation scores. In the MoCA result, $13.5 \%$ of the variance was due to the number of attended sessions, with a power of $97.9 \%$. In the MMSE result, $5 \%$ of the variance was due to the number of attended sessions, with a power of $63.3 \%$ (Table 4 ).

\section{Discussion}

The present study aimed to examine the effects of a combined computer-based and physical activity program on cognitive functions and independence in daily life in community-dwelling elderly individuals. We found a significant positive effect of the intervention on cognitive function, as measured by MoCA and MMSE scores. No significant effects of the intervention were found on ADL and other neuropsychological tests. 
Table 4. Initial and final regression models assessing the effect of the number of attended intervention sessions and control groups on MoCA and MMSE after 4 months.

\begin{tabular}{|c|c|c|c|c|c|c|c|}
\hline $\begin{array}{l}\text { Dependent } \\
\text { variable }\end{array}$ & $\begin{array}{l}\text { Independent } \\
\text { variable }\end{array}$ & B & $\mathrm{Cl} 95 \%$ & $\mathbf{t}$ & $\mathbf{p}$ & $\begin{array}{c}\text { Partial } \eta^{2} \\
(\%)\end{array}$ & $\begin{array}{c}\text { Power } \\
(\%)\end{array}$ \\
\hline Initial model & Constant* & 23.7 & {$[22.3 ; 25.1]$} & 33.2 & $<0.001$ & & \\
\hline \multirow[t]{8}{*}{$\mathrm{MoCA}$} & $\begin{array}{l}\text { Number of } \\
\text { attended sessions }\end{array}$ & 0.09 & {$[0.03 ; 0.15]$} & 3.09 & 0.003 & 8.9 & 86.4 \\
\hline & MoCA baseline ct & 0.6 & {$[0.4 ; 0.7]$} & 7.4 & $<0.001$ & 35.5 & 100.0 \\
\hline & $\begin{array}{l}\text { Change in constant } \\
\text { due to intervention }\end{array}$ & -0.6 & {$[-2.1 ; 0.9]$} & -0.8 & 0.430 & 0.6 & 12.3 \\
\hline & Female gender & 0.9 & {$[-0.3 ; 2.1]$} & 1.5 & 0.137 & 2.2 & 31.7 \\
\hline & Low schooling & -2.3 & {$[-4.9 ; 0.3]$} & -1.8 & 0.079 & 3.1 & 42.1 \\
\hline & Medium schooling & -0.3 & {$[-1.7 ; 1.0]$} & -0.5 & 0.626 & 0.2 & 7.7 \\
\hline & CDR 0.5 & -0.4 & {$[-1.4 ; 0.5]$} & -0.9 & 0.363 & 0.9 & 14.8 \\
\hline & Age ct & -0.03 & {$[-0.10 ; 0.04]$} & -1.0 & 0.337 & 0.9 & 15.9 \\
\hline Final model & Constant* & 23.9 & {$[23.4 ; 24.5]$} & 81.7 & $<0.001$ & & \\
\hline \multirow[t]{2}{*}{ MoCA } & $\begin{array}{c}\text { Number of } \\
\text { attended sessions }\end{array}$ & 0.07 & {$[0.04 ; 0.11]$} & 4.0 & $<0.001$ & 13.5 & 97.9 \\
\hline & MoCA baseline ct & 0.7 & {$[0.5 ; 0.8]$} & 10.1 & $<0.001$ & 49.4 & 100.0 \\
\hline Initial model & Constant* & 27.9 & {$[27.0 ; 28.9]$} & 57.1 & $<0.001$ & & \\
\hline \multirow[t]{8}{*}{ MMSE } & $\begin{array}{c}\text { Number of } \\
\text { attended sessions }\end{array}$ & 0.05 & {$[0.01 ; 0.09]$} & 2.2 & 0.030 & 4.7 & 58.9 \\
\hline & MMSE baseline ct & 0.3 & {$[0.2 ; 0.4]$} & 4.2 & $<0.001$ & 15.0 & 98.4 \\
\hline & $\begin{array}{l}\text { Change in constant } \\
\text { due to intervention }\end{array}$ & -0.5 & {$[-1.5 ; 0.5]$} & -1.0 & 0.336 & 0.9 & 16.0 \\
\hline & Female gender & 0.4 & {$[-0.5 ; 1.2]$} & 0.8 & 0.402 & 0.7 & 13.3 \\
\hline & Low schooling & -1.9 & {$[-3.6 ;-0.2]$} & -2.2 & 0.030 & 4.7 & 58.7 \\
\hline & Medium schooling & -1.7 & {$[-2.6 ;-0.8]$} & -3.7 & $<0.001$ & 12.0 & 95.1 \\
\hline & CDR 0.5 & -0.6 & {$[-1.2 ; 0.1]$} & -1.7 & 0.093 & 2.9 & 39.0 \\
\hline & Age ct & 0.01 & {$[-0.04 ; 0.06]$} & 0.43 & 0.665 & 0.2 & 7.1 \\
\hline Final model & Constant* & 27.9 & {$[27.5 ; 28.4]$} & 126.5 & $<0.001$ & & \\
\hline \multirow[t]{4}{*}{ MMSE } & $\begin{array}{c}\text { Number of } \\
\text { attended sessions }\end{array}$ & 0.03 & {$[0.01 ; 0.05]$} & 2.3 & 0.022 & 5.0 & 63.3 \\
\hline & MMSE baseline ct & 0.3 & {$[0.2 ; 0.4]$} & 4.4 & $<0.001$ & 16.0 & 99.2 \\
\hline & Low schooling & -1.9 & {$[-3.6 ;-0.2]$} & -2.2 & 0.032 & 4.4 & 57.5 \\
\hline & Medium schooling & -1.7 & {$[-2.6 ;-0.8]$} & -3.84 & $<0.001$ & 12.6 & 96.7 \\
\hline
\end{tabular}

*Reference: control group, male gender, high schooling, $\mathrm{ct}=$ centered at baseline mean value. $\mathrm{Cl}$ : confidence interval; MoCA: Montreal Cognitive Assessment; MMSE: Mini-Mental Status Exam; ITT: Intention to treat.

In recent years, the interest in non-pharmacological methods of preventing cognitive and functional decline has increased. In line with the results of the present study, some interventional studies have shown positive results on attention (Rahe et al., 2015) and the global cognitive function of older people (Shatil, 2013). In addition, recent systematic reviews and meta-analysis have shown that combined cognitive and physical ex- ercises have a positive influence on the global cognition of healthy older people (BrudererHofstetter et al., 2018; Lauenroth et al., 2016). A promising result, especially as cognitive decline increases the risk for mild cognitive impairment and further dementia (Prince et al., 2015). Considering the study population, a previous investigation showed that older people with a CDR of 0.5 had 3.82 times higher risk of converting to dementia in relation to CDR 0 (Montaño, An- 
dreoni, \& Ramos, 2013).

Our finding also corroborates results from a study based on the same intervention methodology conducted in Florianopolis, Brazil that demonstrated a significant increase in the MMSE score comparing the intervention group with the control group (Krug et al., 2017).

Likewise, the combination of computerised cognitive exercises to a strength-balance exercise protocol in a randomized controlled trial showed positive interaction effects for dual tasks of walking and divided attention, strengthening the notion that it is advantageous to combine physical and cognitive training in clinical practice (Van het Reve \& de Bruin, 2014).

However, there is a lot of diversity in the design of interventions, and there is no consensus on which features are the most effective. Recent literature reviews point out that aspects such as length (minutes per session), frequency (sessions per week), duration (number of weeks), and intensity of cognitive inclusion influence effectiveness (Bruderer-Hofstetter et al., 2018; Clare \& Woods, 2003; Lampit et al., 2014; Zhu et al., 2016; Laurenroth et al., 2016).

In the present study, we did not observe intervention effects on specific cognitive domains such as memory and verbal fluency. This can be understood by the characteristic of the program that aimed at global cognitive stimulation and did not focus on a specific domain. This result is in line with some other studies that did not demonstrate effects on memory after 16 weeks of combined physical plus cognitive activity focused on the simultaneous training of several basic cognitive abilities (Linde \& Alfermann, 2014).

Considering the results of MMSE and MoCA, it should be noted that, although the MMSE is the most widely used brief cognitive test to monitor change by serial testing in clinical and research settings, it is already known that the two tests have different psychometric properties, with the MoCA showing higher sensitivity for cognitive evaluation (Smith et al. 2007).

The results of the present study indicate the significance of participant's adherence to intervention activities on improved cognitive function. The participants who attended more sessions had greater benefits. The older population is more susceptible to occurrences that may restrict participation in more extensive programs. In our study, the main reasons reported for nonpresence in the intervention were health problems and scheduling incompatibility. Although we phone called all absentees to check why they did not have the last intervention, only $54 \%$ of the participants attended more than $70 \%$ of the program. We did not find any studies in the literature that considered adherence to intervention activities as an independent variable for improved cognitive function. Our study findings suggest that adherence is paramount and a factor to be sought and facilitated in preventive programs for older adults in the community. This aspect could play a crucial role in the design choices for effectiveness and may clarify aspects related to the dose-response of this intervention (Bruderer-Hofstetter et al., 2018).

In addition, the results showed that the educational level was an independent factor in the results of the MMSE and verbal fluency. The relation between MMSE and educational level is already well registered in the literature (Bertolucci, 1994, Crum, 1993). The majority of participants in this study had more than eight years of study (81.1\% in IG and $77.8 \%$ in CG). This factor can have had an influence on the baseline status of the subjects and possible ceiling effects on the measurements taken. In addition, age plays a role in the results for word list and evocation, which include a great component of memory ability, a very sensitive domain in the aging process(La Rue, 2010).

We did not find an intervention effect in independence in activities of daily living. Oswald et al (2006) found a significant improvement in physical function (i.e., index of measures of coordination, flexibility, rhythm, adaptability, readjustment to a moving object, agility/endurance and strength), which is closely related to IADL, after a combined cognitive and physical intervention. Considering that in the present study the endpoint was limitations in $\mathrm{ADL}$, we can understand that the volume and duration of the physical activity component may have been insufficient to generate sizeable benefits, reinforcing the importance of following the guidelines for physical activity for older people for this purpose (Chodzko-Zajko et al., 2009, WHO, 2010). Although some specific cognitive domains are strongly related with instrumental ADLs, especially executive function, working memory, and attention (McAlister \& Schmitter-Edgecombe, 2016), other studies also did not find any effectiveness of combined cognitive and physical interventions to improve IADL (Bruderer-Hofstetter et al., 2018, Fiatarone Singh et al., 2014) reinforcing the need to better understand the underlying mechanisms in this possible association.

The current study had several limitations. First, we had a small sample size, a fact that was shaped by the study's operational characteristics, thereby naturally reducing the statistical power. In addi- 
tion, this limited number of study participants precludes analyses considering healthy individuals (normal cognition) separately from those with mild cognitive impairment $(\mathrm{CDR}=0,5)$, which would allow more accuracy in comparison with the literature. Second, due to operational limitations, we did not properly randomize the participants into treatment groups, thus potentially contributing to the introduction of confounding and selection bias. However, our inclusion criteria based on CDR and frequency matching of the intervention and control groups according to gender and CDR, rendered treatment groups somewhat comparable regarding the main variables of interest in the analysis. The fact that all study participants live in the same middle- to the upper-middle class neighbourhood, and have a very high level of education attainment, also contributed to their similarities regarding outcome and explanatory variables. In addition, we added all participants to the analyses, as an intention to treat is a recommended approach to several types of non-adherence to the study protocol (Petkova \& Teresi, 2002). Third, we did not have a physical capacity-only measurement to verify possible effects on physical functions, or other specific cognitive function tests, to assess executive func- tions and reaction time, assuming that other instruments might have been better at detecting differences. It should also be considered that the study population had a high socioeconomic and educational level, which could have had a celling effect on the intervention results. Finally, it is possible that the control group subjects could have engaged in physical activities and computer use on their own, since recording private activities were not contemplated by the study design. However, this could only bias our results towards the null hypothesis of no association.

\section{Conclusion}

A combined computer-based and physical activity intervention had a positive effect on the global cognition of community-dwelling older adults. This study, however, showed that the intervention did not affect specific cognitive domains or independence in daily life activities. Future research with a larger sample size, longer duration, and blind-randomized design should help increase the understanding of the effectiveness of combined cognitive and physical interventions to prevent cognitive and physical function loss among older people living in the community.

\section{Funding}

This work was supported by the Fundação de Amparo à Pesquisa do Estado de São Paulo(FAPESP) [2014/500849] and the Coordenação de Aperfeiçoamento de Pessoal de Nível Superior(CAPES) [88881.133269/2016-01].

\section{References}

Atkinson, R. C. \& Shiffrin, R. M. (1971). The control of short-memory. Scientific American 225(2) 8290.

Bamidis, P. D., Vivas, A. B., Styliadis, C., Frantzidis, C., Klados, M., Schlee, W., Papageorgiou, S. G. (2014). A review of physical and cognitive interventions in aging. Neuroscience \& Biobehavioral Reviews, 44, 206-220. https://doi.org/10.1016/j.neubiorev.2014.03.019

Bertolucci, P. H.F., Brucki, S. M.D., Campacci, S. R., \& Juliano, Y. (1994). O Mini-Exame do Estado Mental em uma população geral: impacto da escolaridade. Arquivos de Neuro-Psiquiatria, 52(1), 01-07. https:// dx.doi.org/10.1590/S0004-282X1994000100001 (in portuguese)

Boz, H., \& Karatas, S. E. (2015). A Review on Internet Use and Quality of Life of the Elderly. Cypriot Journal of Educational Sciences, 10(3), 182-191.

Bruderer-Hofstetter, M., Rausch-Osthoff, A.-K., Meichtry, A., Münzer, T., \& Niedermann, K. (2018). Effective multicomponent interventions in comparison to active control and no interventions on physical capacity, cognitive function and instrumental activities of daily living in elderly people with and without mild impaired cognition - A systematic review and network meta-analysis. Ageing Research Reviews, 45, 1-14. https://doi.org/10.1016/j. arr.2018.04.002

Chodzko-Zajko, W. J., Proctor, D. N., Singh, M. A. F., Minson, C. T., Nigg, C. R., Salem, G. J., \& Skinner, J. S. (2009). Exercise and physical activity for older adults. Medicine \& science in sports \& exercise, 41(7), 1510-1530.

Clare, L., \& Woods, B. (2003). Cognitive rehabilitation and cognitive training for early-stage Alzheimer's disease and vascular dementia. In The Cochrane Collaboration (Ed.), Cochrane Database of Systematic Reviews. Chichester, UK: John Wiley \& Sons, Ltd. https://doi.org/10.1002/14651858. CD003260

Crum, R. M. (1993). Population-Based Norms for the Mini-Mental State Examination by Age and Educational Level, 6.

D'Orsi, E., Xavier, A. J., Steptoe, A., de Oliveira, C., Ramos, L. R., Orrell, M., Marmot, M. G. (2014). Socioeconomic and Lifestyle Factors Related to Instrumental Activity of Daily Living Dynamics: Results from the English Longitudinal Study of Ageing. Journal of the American Geriatrics Society, 62(9), 1630-1639. https://doi.org/10.1111/jgs.12990

D'Orsi, E., Xavier, A. J., Rafnsson, S. B., Steptoe, A., Hogervorst, E., \& Orrell, M. (2017). Is use of the internet in midlife associated with lower dementia incidence? Results from the English Longitudinal Study of Ageing. Aging \& Mental Health, 1-9. https://doi.org/10.1080/13607863.2017.1360840

Fiatarone Singh, M. A., Gates, N., Saigal, N., Wilson, G. C., Meiklejohn, J., Brodaty, H., Valenzuela, M. (2014). The Study of Mental and Resistance Training (SMART) Study-Resistance Training and/or Cognitive Training in Mild Cognitive Impairment: A Randomized, Double-Blind, Double-Sham Con- 


\section{Digital inclusion, physical activity, and cognition}

trolled Trial. Journal of the American Medical Directors Association, 15(12), 873-880. https://doi. org/10.1016/j.jamda.2014.09.010

Folstein, M. F., Folstein, S. E., \& McHugh, P. R. (1975). Mini-mental state. Journal of Psychiatric Research, 12(3), 189-198. https://doi.org/10.1016/00223956(75)90026-6

Isaacs, B., \& Kennie, A. T. (1973). The Set Test as an Aid to the Detection of Dementia in Old People. The British Journal of Psychiatry, 123(4), 467-470. https://doi.org/10.1192/bjp.123.4.467

Gates NJ, Rutjes AWS, Di Nisio M, Karim S, Chong LY, March E, Martínez G, Vernooij RWM. Computerised cognitive training for maintaining cognitive function in cognitively healthy people in late life. (2019). Cochrane Database of Systematic Reviews. 3: CD012277. https://doi.org/10.1002/14651858. CD012277.pub2.

Klusmann, V., Evers, A., Schwarzer, R., Schlattmann, P., Reischies, F. M., Heuser, I., \& Dimeo, F. C. (2010). Complex Mental and Physical Activity in Older Women and Cognitive Performance: A 6-month Randomized Controlled Trial. The Journals of Gerontology Series A: Biological Sciences and Medical Sciences, 65A(6), 680-688. https://doi. org/10.1093/gerona/glq053

Krug, R., Ono, L., Quialheiro, A., D'Orsi, E., Ramos, L., \& Xavier, A. (2015). A Stimulation and rehabilitation program: Oficina da Lembrança. Revista Brasileira de Atividade Física \& Saúde, 20(5), 534. https://doi.org/10.12820/rbafs.v.20n5p534

Krug, R. de R., Silva, A. Q. A. da, Schneider, I. J. C., Ramos, L. R., d'Orsi, E., \& Xavier, A. J. (2017). Cognitive cooperation groups mediated by computers and internet present significant improvement of cognitive status in older adults with memory complaints: a controlled prospective study. Arquivos de Neuro-Psiquiatria, 75(4), 228-233. https://doi. org/10.1590/0004-282×20170021

Kueider, A. M., Parisi, J. M., Gross, A. L., \& Rebok, G. W. (2012). Computerized Cognitive Training with Older Adults: A Systematic Review. PLoS ONE, 7(7), e40588. https://doi.org/10.1371/journal. pone.0040588

La Rue, A. (2010). Healthy Brain Aging: Role of Cognitive Reserve, Cognitive Stimulation, and Cognitive Exercises. Clinics in Geriatric Medicine, 26(1), 99111. https://doi.org/10.1016/j.cger.2009.11.003

Lampit, A., Hallock, H., \& Valenzuela, M. (2014). Computerized Cognitive Training in Cognitively Healthy Older Adults: A Systematic Review and Meta-Analysis of Effect Modifiers. PLoS Medicine, 11(11), e1001756. https://doi.org/10.1371/journal. pmed.1001756

Lankshear, C., \& Knobel, M. (2008). Introduction: Digital literacies-Concepts, policies and practices. In C. Lankshear \& M. Knobel (Eds.), Digital literacies: Concepts, policies and practices (pp. 1-16). New York, NY: Peter Lang

Laurenroth, A., Ioannidis, A. E., \& Teichmann, B. (2016). Influence of combined physical and cognitive training on cognition: a systematic review. BMC Geriatrics, 16(1). https://doi.org/10.1186/s12877016-0315-1
Law, L. L., Barnett, F., Yau, M. K., \& Gray, M. A. (2014). Effects of combined cognitive and exercise interventions on cognition in older adults with and without cognitive impairment: a systematic review. Ageing research reviews, 15, 61-75.

Linde, K., \& Alfermann, D. (2014). Single versus Combined Cognitive and Physical Activity Effects on Fluid Cognitive Abilities of Healthy Older Adults: A 4-Month Randomized Controlled Trial with FollowUp. Journal of Aging and Physical Activity, 22(3), 302-313. https://doi.org/10.1123/JAPA.2012-0149

Livingston, G., Sommerlad, A., Orgeta, V., Costafreda, S. G., Huntley, J., Ames, D., \& Cooper, C. (2017). Dementia prevention, intervention, and care. The Lancet, 390(10113), 2673-2734

Martin, M., Clare, L., Altgassen, A. M., Cameron, M. H., \& Zehnder, F. (2011). Cognition-based interventions for healthy older people and people with mild cognitive impairment. Cochrane Database of Systematic Reviews. https://doi.org/10.1002/14651858. CD006220.pub2

McAlister, C., \& Schmitter-Edgecombe, M. (2016). Executive function subcomponents and their relations to everyday functioning in healthy older adults. Journal of Clinical and Experimental Neuropsychology, 38(8), 925-940. https://doi. org/10.1080/13803395.2016. 1177490

Montaño, M. B. M., \& Ramos, L. R. (2005). Validade da versão em português da Clinical Dementia Rating. Revista de Saúde Pública, 39(6), 912-917. https:// doi.org/10.1590/S0034-89102005000600007

Montaño, M. B. M., Andreoni, S., \& Ramos, L. R. (2013). Clinical Dementia Rating independently predicted conversion to dementia in a cohort of urban elderly in Brazil. International Psychogeriatrics, 25(2), 245 251.

Morris, J. (1996). Clinical Dementia Rating: A Reliable and Valid Diagnostic and Staging Measure for Dementia of the Alzheimer Type. International Psychogeriatrics (supS1) 1997:173-176.

Nasreddine, Z. S., Phillips, N. A., Bédirian, V., Charbonneau, S., Whitehead, V., Collin, I., \& Chertkow, H. (2005). The Montreal Cognitive Assessment, MoCA: a brief screening tool for mild cognitive impairment. Journal of the American Geriatrics Society, 53(4), 695-699.

Nitrini, R., Bottino, C. M. C., Albala, C., Custodio Capuñay, N. S., Ketzoian, C., Llibre Rodriguez, Caramelli, P. (2009). Prevalence of dementia in Latin America: a collaborative study of population-based cohorts. International Psychogeriatrics, 21(04), 622. https:// doi.org/10.1017/S1041610209009430

Oswald W, Gunzelmann T, Rupprecht R, Hagen B. (2006). Differential effects of single versus combined cognitive and physical training with older adults: The SimA study in a 5-year perspective. Eur. J. Ageing 3, 179-192

Paradela, E. M. P., Lourenço, R. A., \& Veras, R. P. (2005). Validation of geriatric depression scale in a general outpatient clinic. Rev Saúde Pública, 39(6):918-923.

Petkova E, Teresi J. (2002). Some statistical issues in the analyses of data from longitudinal studies of elderly chronic care populations. Psychosom Med 64(3):531-547. 


\section{Digital inclusion, physical activity, and cognition}

Prince, M ., Albanese, E., Guerchet, M., \& Prina, M. (2014). World Alzheimer Report 2014. Dementia and Risk Reduction: an analysis of protective and modifiable factors. https://www.alz.co.uk/research/WorldAlzheimerReport2014.pdf

Prince, M., Wimo, A., Guerchet, M., Ali, G. C., Wu, Y. T., \& Prina, M. (2015). World Alzheimer Report 2015-The Global Impact of Dementia: An analysis of prevalence, incidence, cost and trends. 2015. accessed in https://www.alz.co.uk/research/worldreport-2015 in 092018

Rahe, J., Petrelli, A., Kaesberg, S., Fink, G. R., Kessler, J., \& Kalbe, E. (2015). Effects of cognitive training with additional physical activity compared to pure cognitive training in healthy older adults. Clinical interventions in aging, 10, 297.

Ramos, L. R., Simoes, E. J., \& Albert, M. S. (2001). Dependence in Activities of Daily Living and Cognitive Impairment Strongly Predicted Mortality in Older Urban Residents in Brazil: A 2-Year FollowUp. Journal of the American Geriatrics Society, 49(9), 1168-1175. https://doi.org/10.1046/j.15325415.2001.49233.x

Ramos, L.R. (2003). Fatores determinantes do envelhecimento saudável em idosos residentes em centro urbano: Projeto Epidoso, São Paulo. Cadernos de Saúde Pública, 19(3), 793-797. https://doi. org/10.1590/S0102-311X2003000300011

Ramos, L. R., Andreoni, S., Coelho-Filho, J. M., LimaCosta, M. F., Matos, D. L., Rebouças, M., \& Veras, R. (2013). Perguntas mínimas para rastrear dependência em atividades da vida diária em idosos. Revista de Saúde Pública, 47(3), 506-513. https://doi. org/10.1590/S0034-8910.2013047004325

Scazufca, M., Menezes, P. R., Araya, R., Di Rienzo, V. D., Almeida, O. P., Gunnell, D., \& Lawlor, D. A. (2008). Risk factors across the life course and dementia in a Brazilian population: results from the Sao Paulo Ageing \& Health Study (SPAH). International Journal of Epidemiology, 37(4), 879-890. https://doi.org/10.1093/ije/dyn125

Shatil, E. (2013). Does combined cognitive training and physical activity training enhance cognitive abilities more than either alone? A four-condition randomized controlled trial among healthy older adults. Front Aging Neurosci. 26;5:8. https://doi. org/10.3389/fnagi.2013.00008.

Shao, Y., Mang, J., Li, P., Wang, J., Deng, T., \& Xu, Z. (2015). Computer-Based Cognitive Programs for Improvement of Memory, Processing Speed and Executive Function during Age-Related Cognitive Decline: A Meta-Analysis. PLOS ONE, 10(6), e0130831. https://doi.org/10.1371/journal. pone.0130831
Singh-Manoux, A., Kivimaki, M., Glymour, M. M. Elbaz, A., Berr, C., Ebmeier, K. P., Dugravot, A. (2012). Timing of onset of cognitive decline: results from Whitehall II prospective cohort study. BMJ, 344(jan04 4), d7622-d7622. https://doi. org/10.1136/bmj.d7622

Small, B. J., \& Bäckman, L. (1999). Time to Death and Cognitive Performance. Current Directions in Psychological Science, 8(6), 168-172. https://doi. org/10.1111/1467-8721.00040

Smith, T; Gilden, N.; Holmes, C. (2007). The Montreal Cognitive Assessment: validity and utility in a memory clinic setting. The Canadian Journal of Psychiatry, v. 52, n. 5, p. 329-332.

Van Het Reve, E., \& de Bruin, E. D. (2014). Strength-balance supplemented with computerized cognitive training to improve dual task gait and divided attention in older adults: a multicenter randomizedcontrolled trial. BMC geriatrics, 14(1), 134.

World Health Organization. (2010). Global recommendations on physical activity for health. World Health Organization.Geneva

Xavier, A, Sales, M., Ramos, L., Anção, M., Sigulem, D. (2004). Cognition, interaction and ageing: an Internet workshops exploratory Study. Studies in Health Technology and Informatics. Ebook 103:289 295: Medical and Care Compunetics. https://doi. org/10.3233/978-1-60750-946-2-289

Xavier, A. J., d'Orsi, E., de Oliveira, C. M., Orrell, M., Demakakos, P., Biddulph, J. P., \& Marmot, M. G.(2014). English Longitudinal Study of Aging: Can Internet/E-mail Use Reduce Cognitive Decline? The Journals of Gerontology Series A: Biological Sciences and aq Medical Sciences, 69(9), 1117-1121. https://doi.org/10.1093/gerona/glu105

Xavier, A. J., D'Orsi, E., Wardle, J., Demakakos, P., Smith, S. G., \& von Wagner, C. (2013). Internet Use and Cancer-Preventive Behaviors in Older Adults: Findings from a Longitudinal Cohort Study. Cancer Epidemiology Biomarkers \& Prevention, 22(11), 2066-2074. https://doi.org/10.1158/1055-9965.EPI$13-0542$

Yesavage, J. A., Brink, T. L., Rose, T. L., Lum, O., Huang, V., Adey, M., \& Leirer, V. O. (1982). Development and validation of a geriatric depression screening scale: A preliminary report. Journal of Psychiatric Research, 17, 37-49. https://doi.org/10.1016/00223956(82)90033-4

Zhu, X., Yin, S., Lang, M., He, R., \& Li, J. (2016). The more the better? A meta-analysis on effects of combined cognitive and physical intervention on cognition in healthy older adults. Ageing Research Reviews, 31, 67-79. https://doi.org/10.1016/j. arr.2016.07.003 